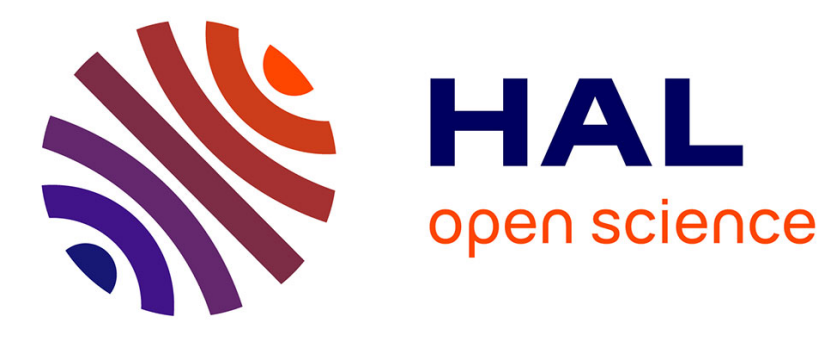

\title{
Stochastic Rotation Dynamics simulation of electro-osmosis
}

Davide R. Ceratti, Amaël Obliger, Marie Jardat, Benjamin Rotenberg, Vincent Dahirel

\section{- To cite this version:}

Davide R. Ceratti, Amaël Obliger, Marie Jardat, Benjamin Rotenberg, Vincent Dahirel. Stochastic Rotation Dynamics simulation of electro-osmosis. Molecular Physics, 2015, pp.1-11. 10.1080/00268976.2015.1037370 . hal-01212122

HAL Id: hal-01212122

https://hal.sorbonne-universite.fr/hal-01212122

Submitted on 6 Oct 2015

HAL is a multi-disciplinary open access archive for the deposit and dissemination of scientific research documents, whether they are published or not. The documents may come from teaching and research institutions in France or abroad, or from public or private research centers.
L'archive ouverte pluridisciplinaire HAL, est destinée au dépôt et à la diffusion de documents scientifiques de niveau recherche, publiés ou non, émanant des établissements d'enseignement et de recherche français ou étrangers, des laboratoires publics ou privés. 
To appear in Molecular Physics

Vol. 00, No. 00, Month 200x, 2-19

\title{
Stochastic Rotation Dynamics simulation of electro-osmosis
}

\author{
Davide R. Ceratti ${ }^{a, b, c}$, Amaël Obliger ${ }^{d}$, Marie Jardat ${ }^{a, b}$, Benjamin Rotenberg $^{a, b}$, Vincent \\ Dahirel $^{a, b *}$ \\ ${ }^{a}$ Sorbonne Universités, UPMC Univ Paris 06, UMR PHENIX, F-75005, Paris, France; \\ ${ }^{b}$ CNRS, UMR PHENIX, F-75005, Paris, France; \\ ${ }^{c}$ Collège de France, UMR 7574, Chimie de la Matière Condensée de Paris; \\ ${ }^{d}$ Concrete Sustainability Hub, Department of Civil and Environmental Engineering, and \\ MIT-CNRS Joint Laboratory, Massachusetts Institute of Technology, 77 Massachusetts \\ Avenue, Cambridge, Massachusetts 02139, United States \\ (Received 00 Month 200x; final version received 00 Month 200x)
}

\begin{abstract}
Stochastic Rotation Dynamics (SRD) is a mesoscale simulation technique that captures hydrodynamic couplings in simple and complex fluids. It can be used in various hydrodynamic regimes and it is not restricted to specific geometries. We show here that SRD using the collisional coupling approach to capture momentum transfer between the semi-implicit solvent and the explicit counterions, is able to describe electro-kinetic effects, i.e. coupled electrostatic and hydrodynamic phenomena occuring at charged solid-liquid interfaces. The method is first validated for electro-osmosis in the simple case of a slit pore without added salt, for which an analytical solution of the Helmholtz-Smoluchowski theory is known, in a physical regime where this mean-field theory is valid. We then discuss the predictions of SRD for electro-osmosis beyond the range of validity of the Helmholtz-Smoluchowski (or Poisson-Nernst-Planck) theory, in particular due to ion-ion correlations at the surface, to charge localization on discrete sites at the solid surface and to surface charge heterogeneity, that all contribute to a reduction of the electro-osmotic flow. In order to disentangle these last two aspects, we also investigate at the mean-field level a simple system with alternate charged and neutral stripes, using LatticeBoltzmann Electrokinetics simulations. Overall, this work opens new perspectives for the use of SRD as a generic mesoscopic simulation method for soft matter problems, in particular under confinement, since in practice many interfaces between fluids and solids are charged.
\end{abstract}

\section{Introduction}

Stochastic Rotation Dynamics (SRD), also known as Multiparticle Collision Dynamics (MPCD), is a mesoscale simulation technique that captures hydrodynamic couplings in simple and complex fluids, in various hydrodynamic regimes and not restricted to specific geometries $[1,2]$. This flexibility, together with the possibility to choose the simulation parameters taylored to specific fluid properties and flow regimes thanks to analytical expressions for the transport coefficients [37], has fostered the development of this approach and its application to a variety of soft matter systems. From simple fluids, the interest rapidly shifted to colloidal suspensions [8-10], polymer or star-polymer solutions [11] micellar systems [12],vesicles [13] or viral capsid self-assembly [14]. For most applications involving soft matter, the ability to describe flow in confined geometries is essential. Therefore, particular attention has been devoted to the derivation of appropriate SRD rules to account for the desired boundary conditions at solid/fluid interfaces [15-17]. It is then possible to simulate complex systems such as the deposition

\footnotetext{
*Corresponding author. Email: vincent.dahirel@upmc.fr
} 
of asphaltene in capillaries [18, 19], complex porous media with nanopillars [20] or even red blood cells in narrow capillaries [21]. Recently, a GPU accelerated version of SRD has been proposed, an improvement which is valudable for large and complex systems for which SRD might get computationaly expensive [22].

In practice, solid surfaces - either confining walls or simply colloidal particles are often charged. The compensating counterions are located in the fluid, in the so-called Debye layer arising from the balance between the electrostatic attraction to the surface and the entropic gain of being in the fluid phase. These charged interfaces are at the origin of electrokinetic effects, i.e. coupled hydrodynamic and electric phenomena. Due to their importance in a variety of contexts, from the environment to biology, or from microfluidics to electroacoustic exploration, various simulation strategies have been developed to address the challenge of the multiple length and time scales involved in electrokinetic phenomena, as discussed in two recent reviews $[23,24]$.

Despite the success of SRD, to date, only few applications to electrokinetic phenomena have been reported, none of which concern the electro-osmosis process. A few works have been devoted to electrophoresis of polyelectrolytes and their counterions $[25,26]$. Some of us have recently demonstrated the possibility of studying the influence of concentration on the transport properties of electrolytes coupling SRD with charged solutes [27]. The proposed hybrid approach, similar to previous works using a Lattice Boltzmann description of the underlying fluid [28-30], couples the SRD fluid with explicit solutes, not only for the colloidal particles but also for microions (as opposed to charged colloidal particles). The semi-implicit fluid allows to capture hydrodynamic interactions between solutes at a moderate simulation cost, while electrostatic correlations between charged solutes are explicitely taken into account. The challenge is then to describe the coupling between solvent and solutes. Several coupling schemes were investigated, and their effect on the solute diffusion coefficient and the electrical conductivity of the solution was discussed [27].

In the present work, we report on the first application of SRD to the case of electro-osmosis, i.e. the fluid flow induced by an applied electric field at the boundary with a fixed charged wall. We investigate the effect of the treatment of ion-wall interactions within the SRD scheme and of the charge distribution at the solid surface on the electro-osmotic flow. In order to validate this new method, we study here a simple slit pore in the absence of added salt and compare with the analytical results of Poisson-Nernst-Planck (PNP) theory, which applies when electrostatic correlations between ions can be neglected. For a homogeneously charged surface, results are compared with the analytical solution of PNP, while for more complex charge distributions simulations using a previously validated Lattice Boltzmann scheme [31-34] (valid under the same conditions as PNP) are used as a reference. Section 2 introduces the electro-osmosis phenomenon, the system chosen to benchmark its description by SRD and the reference analytical model. The simuation methodology is presented in detail in Section 3. Finally, simulation results are presented and discussed in Section 4.

\section{Electro-osmosis and SRD}

\subsection{Electro-osmosis}

Electro-osmosis is a phenomenon occuring when an electric field is applied to a fluid in the vicinity of a charged surface. The local electric charge in the doublelayer results in a local electric force accelerating the fluid. The fluid is usually 
charge neutral far from the surfaces (beyond the so-called Debye length, of the order of $1 \mathrm{~nm}$ for typical electrolyte concentrations in water at room temperature). Nevertheless, momentum diffusion through the viscous fluid results in a flow even in the neutral regions.

The standard description of electro-osmosis is provided, at the mean-field level, by the Poisson-Nernst-Planck (PNP) theory, which combines: (i) the Navier-Stokes equation for momentum conservation within the fluid, including the local electric force, (ii) the Nernst-Planck equation for the ionic fluxes due to local electrochemical potential gradients and (iii) the Poisson equation relating the electrostatic potential to the ionic concentrations. At steady state and for small Reynolds numbers, the Navier-Stokes equation reduces to Stokes equation: $\eta \Delta \mathbf{u}+\mathbf{F}_{\mathbf{v}}=\mathbf{0}$, where $\mathbf{u}$ is the fluid velocity, $\eta$ its viscosity and $\mathbf{F}_{\mathbf{v}}$ the volumic force acting on it. Similarly, the Nernst-Planck and Poisson equations converge to the Poisson-Boltzmann (PB) solution. The Stokes and PB equations are the basis of the so-called HelmhotlzSmoluchowski theory, which will be presented below.

\subsection{Benchmarking SRD against an analytical continuous theory}

Although in principle Stochastic Rotation Dynamics accounts for hydrodynamical effects at the same level as the Navier-Stokes equation, using this simulation methodology in prescribed hydrodynamic regimes is not straightforward. The effect of thermal fluctuations and that of the discreteness of the solvent and solutes, as well as the the finite value of dimensionless hydrodynamic numbers can lead to practical difficulties. As an example, significantly reducing or increasing the Reynolds, Schmidt or Peclet numbers is only possible at the price of considerably increasing the computational cost. Therefore, some ambiguity may remain when comparing the SRD fluid to a real one in a well-defined hydrodynamic regime. Moreover, as pointed out in the introduction, even if the behaviour of the pure solvent is perfectly controlled, coupling the solvent to a particle or a solid surface always leads to additional approximations and a trade-off between efficiency and accuracy.

For all these reasons, one should benchmark this technique against analytical theories for systems where the theory is known to work. The present study of electro-osmosis can thus be viewed in the broader perspective of the validation of the SRD method. One of the few cases for which the PNP model can be solved analytically for the electro-osmosis problem is that of a charged slit pore, i.e. two infinite parallel walls, in the absence of added salt. While this solution is exact, it suffers from the limitation of the underlying PNP theory. Fortunately, its range of validity is rather well established. As a mean-field continuous theory, it neglects ionic correlations (both electrostatic and due to their finite size) and is thus limited to sufficiently small local ionic densities. In particular, it is expected to break down for large surface charge densities, with multivalent ions or too small distances between the surfaces. In addition, the Helmholtz-Smoluchowski theory is limited to the laminar regime of small Reynolds numbers. We will thus benchmark the proposed SRD method in this very simple geometry and under the above constraints. Once the method is validated, we can examine the predictions of SRD beyond the range of validity of the PNP model.

\subsection{Poisson-Nernst-Planck / Helmholtz-Smoluchowski theory}

In the case of electro-osmosis, the volumic force $\mathbf{F}_{\mathbf{v}}$ entering the Stokes equation to balance the viscous stress arises from an applied electric field $\mathbf{E}=E_{x} \mathbf{e}_{\mathbf{x}}$ along 
the surfaces, i.e. $\mathbf{F}_{\mathbf{v}}=c_{+}(z) e \mathbf{E}$, where $c_{+}(z)$ is the local counterion concentration. The solution for the velocity profile $u_{x}(z)$ in the laminar regime reads:

$$
u_{x}(z)=u_{x}\left(L_{\mathrm{hyd}} / 2\right)-\frac{e E}{\eta} \int_{L_{\mathrm{hyd}} / 2}^{z} \int_{0}^{z^{\prime}} c_{+}\left(z^{\prime \prime}\right) \mathrm{d} z^{\prime \prime} \mathrm{d} z^{\prime}
$$

where $u_{x}\left(L_{\mathrm{hyd}} / 2\right)$ is determined by the boundary conditions. The ionic density profile can be determined either from a simulation (Molecular Dynamics or in the present case SRD) or from a continuous theory such as Poisson-Boltzmann.

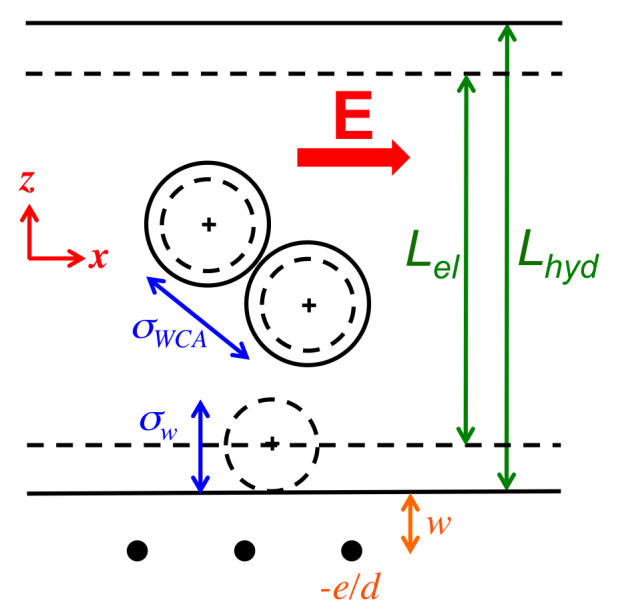

Figure 1. System simulated with Stochastic Rotation Dynamics. Two parallel, infinite charged walls are separated by counterions in a solvent. An electric field $E$ is applied along the surfaces. The solvent can explore the whole space between the walls, separated by a distance $L_{h y d}$, while an effective ion-wall diameter $\sigma_{w}$ restricts the ion to a space of width $L_{e l}=L_{h y d}-\sigma_{w}$. The average surface charge density $\sigma e$ is distributed over discrete size of charge $-e / d$ located at a depth $w$ within the solid wall. The short-range repulsion between ions may be taken into account by a WCA potential, with diameter $\sigma_{W C A}$.

In order to account for the finite size of the ions, the PB equation should be solved over a domain of width $L_{e l}=L_{h y d}-\sigma_{w}$, with $L_{h y d}$ the distance between the walls and $\sigma_{w}$ an effective exclusion diameter of the ions from the wall (see Fig. 1). Denoting $\sigma e$ the homogeneous surface charge density, with $e$ the elementary charge and $\sigma<0$, the counterion density profile for $z \in\left[-L_{\mathrm{el}} / 2,+L_{\mathrm{el}} / 2\right]$ can be computed analytically. The solution reads (see e.g. [35]): $c_{+}(z)=\frac{\alpha^{2}}{2 \pi l_{B}} \frac{1}{\cos ^{2} \alpha z}$ where $\frac{\alpha L_{\mathrm{el}}}{2} \tan \frac{\alpha L_{\mathrm{el}}}{2}=\pi \sigma L_{\mathrm{el}} l_{B}$, is $l_{B}=e^{2} / 4 \pi \epsilon_{0} \epsilon_{r} k_{B} T$ is the Bjerrum length $\left(\epsilon_{r}\right.$ is the solvent permittivity, $k_{B}$ is Boltzmann's constant and $T$ the temperature), while $\alpha^{-1}$ stands for the analog of the Debye screening length and characterizes the distorsion of the counterion profile. For small charge densities, $\alpha L_{\mathrm{el}} \sim \sqrt{4 \pi \sigma L_{\mathrm{el}} l_{B}}$, whereas at large charge densities $\alpha L_{\mathrm{el}} \rightarrow \pi$.

When the effective exclusion diameter of the ions $\sigma_{w}$ vanishes $\left(L_{\mathrm{el}}=L_{\mathrm{hyd}}=L\right)$, the electro-osmotic flow in the presence of an applied electric field $E_{x} \mathbf{e}_{\mathbf{x}}$ is computed by injecting the $\mathrm{PB}$ concentration profile in Eq. 1, with the result:

$$
u_{x}(z)=\frac{e E_{x}}{2 \pi \eta l_{B}} \ln \frac{\cos \alpha z}{\cos \alpha L / 2} \equiv v^{\circ} h(z)
$$

with a characteristic velocity $v^{\circ}=e E_{y} / 2 \pi \eta l_{B}$. This characteristic velocity can be 
used to compute the Reynolds number:

$$
R e=\frac{v^{\circ} L_{\mathrm{hyd}} \rho}{\eta}
$$

In the general case of $\sigma_{w} \neq 0$ the solvent velocity profile is linear between $-L_{\mathrm{hyd}} / 2$ and $-L_{\mathrm{el}} / 2$, with a slope equal to $\sigma e E / \eta$. In the following, we consider the case of water at room temperature, leading to $l_{B}=0.714 \mathrm{~nm}$, and a fixed distance between the walls $L_{\mathrm{hyd}}=4.5 \mathrm{~nm}$.

\section{Methods}

\subsection{Stochastic Rotation Dynamics}

\subsubsection{Pure SRD fluid}

In SRD simulations, the fluid is represented by pointlike particles, whose positions and velocities evolve in two steps. First, in the streaming step, positions and velocities are propagated by integrating Newton's equations of motion, for a time denoted by $\delta t_{c}$. In the absence of external forces, this yields a ballistic motion for each fluid particle $i$ :

$$
\mathbf{r}_{i}\left(t+\delta t_{c}\right)=\mathbf{r}_{i}(t)+\mathbf{v}_{i}(t) \delta t_{c}
$$

where $\mathbf{r}_{i}, \mathbf{v}_{i}$ are the position and the velocity of particle $i$, respectively. Second, in the collision step, the simulation box is divided into cubic cells of size $a_{0}$, where momentum exchanges between the enclosed fluid particles occur. More precisely, a randomly oriented axis is defined for each collision cell, and the velocities of fluid particles relative to the velocity $\mathbf{v}_{\text {c.o.m }}^{\text {cell }}$ of the center of mass of the cell are rotated by an angle $\alpha$ around this axis:

$$
\mathbf{v}_{i}\left(t+\delta t_{c}\right)=\mathbf{v}_{c . o . m}^{c e l l}(t)+\mathcal{R}_{\alpha}\left[\mathbf{v}_{i}(t)-\mathbf{v}_{c . o . m}^{\text {cell }}(t)\right]
$$

where $\mathcal{R}_{\alpha}$ is the rotation matrix. The angle $\alpha$ is a fixed parameter of the simulation. The collision step conserves the velocity of the center-of-mass of the cell. To ensure the galilean invariance, a random shift of the collision grid has to be performed at each collision step [3, 36].

This algorithm ensures the conservation of the local momentum of the fluid, while enabling momentum tranfers inside the fluid. The SRD fluid has thus the hydrodynamic characteristics of a real fluid, in particular in terms of dimensionless hydrodynamic numbers, as discussed in detail by Padding and Louis [9]. The amount of momentum that is exchanged in a cell per unit time, and thus the viscosity of the SRD fluid, depends on the number of solvent particles per cell $\gamma$, on the rotation angle $\alpha$, and on the time interval between collision steps $\delta t_{c}$. The simplicity of the SRD algorithm makes possible the derivation of analytical formulas for the viscosity and transport coefficients as functions of $\gamma, \alpha$, and $\delta t_{c}[3-7]$. In what follows, we use dimensionless units depending on the fluid particle mass $m_{f}$ as the mass unit, the size of the collision cells $a_{0}$ as the length unit, and $k_{B} T$ as the energy unit with $T$ the temperature and $k_{B}$ the Boltzmann constant. The time unit is then

$$
t_{0}=a_{0} \sqrt{\frac{m_{f}}{k_{B} T}}
$$


The value of the collision time step $\delta t_{c}$ controls the fluid properties by modulating the dimensionless mean free path $\lambda$ of fluid particles defined by $\lambda=\delta t_{c} / t_{0}[9]$.

\subsubsection{Embedded solute particles in a SRD bath: Collisional Coupling}

The simplest method to achieve the coupling between solute particles and the fluid bath is to treat the solutes like fluid particles, except that they are interacting with each other, and may have a larger mass than that of fluid particles. In the streaming step, the positions of fluid particles are updated following Eq. (4), whereas the position and velocity of solutes, denoted respectively by $\mathbf{R}_{j}$ and $\mathbf{V}_{j}$ for solute $j$, are propagated thanks to the velocity Verlet algorithm of standard Molecular Dynamics (MD) simulations, with a time step $\delta t_{M D}$ :

$$
\begin{gathered}
\mathbf{R}_{j}\left(t+\delta t_{M D}\right)=\mathbf{R}_{j}(t)+\mathbf{V}_{j}(t) \delta t_{M D}+\frac{\mathbf{F}_{j}(t)}{2 M} \delta t_{M D}^{2}, \\
\mathbf{V}_{j}\left(t+\delta t_{M D}\right)=\mathbf{V}_{j}(t)+\frac{\mathbf{F}_{j}(t)+\mathbf{F}_{j}\left(t+\delta t_{M D}\right)}{2 M} \delta t_{M D},
\end{gathered}
$$

where $M$ is the solute mass and $\mathbf{F}_{j}$ is the force acting on solute $j$ at the beginning of the step, which derives from a given interaction potential. Like in standard MD, the choice of $\delta t_{M D}$ relies on a compromise between numerical efficiency and precision. The value of the time step $\delta t_{M D}$ depends then on the nature of the interaction potential, and is often smaller than $\delta t_{c}$. In the collision step, the velocities of each particle, fluid and solute, included in each collision cell are updated following Eq. (5) given above. This momentum exchange is the only interaction between fluid and solute particles in this simulation scheme. Details of this simulation scheme can be found in Refs [37, 38].

\subsubsection{Simulation of a slit pore}

The simulation box is limited in the $z$-direction by two infinite charged walls separated by a distance $L_{\text {hyd }}$. Periodic boundary conditions are applied along the $x, y$ directions. We apply a non-slip boundary condition between the solvent and the walls by using the bounce-back reflection rules and the so-called virtual particles method introduced by Lamura et al. [39]. In order to account for the size of the counterions, we also use the bounce-back rules for the ions, but at a finite distance from the wall. Ions are thus treated as hard spheres with a non-slip boundary condition at the wall. A thermostat at the cell level is used to ensure constant temperature [38]. Discrete charges are put on the wall at a short depth $w$ within the wall (see Fig. 1 and next section). Finally, the electro-osmotic flow is induced by applying an electric field along the $x$-direction.

\subsubsection{Ion-ion and ion-wall interactions}

As already stated, ions are treated as hard spheres with regard to the wall. Moreover, Coulomb interactions are taken into account between ions $i$ and $j$ and between ions and charged sites of the walls:

$$
U_{e l}\left(r_{i j}\right)=k_{B} T \frac{l_{B} z_{i} z_{j}}{r_{i j}},
$$

with $z_{i}$ the valency of charge $i$ and $r_{i j}$ the distance between $i$ and $j$. Long-range Coulomb interactions are computed using Ewald summation following the method of $[40]$. 
In most of our simulations, no other interactions between ions are introduced, so that it is not needed to split the streaming step into smaller MD steps, as the potential is smooth enough $\left(\delta t_{M D}=\delta t_{c}\right)$. Note that this is possible because only counterions are present, otherwise the attraction between oppositely charged ions should be balanced by a short-range repulsion. Nevertheless, in order to assess the influence of excluded volume effects between ions, we have in some cases also added a WCA (Weeks-Chandler-Anderson) potential [41], which is purely repulsive:

$$
U_{W C A}\left(r_{i j}\right)=\left\{\begin{array}{c}
4 \epsilon_{W C A}\left[\left(\frac{\sigma_{W C A}}{r_{i j}}\right)^{12}-\left(\frac{\sigma_{W C A}}{r_{i j}}\right)^{6}\right]+\epsilon \text { if } r_{i j}<2^{1 / 6} \sigma_{W C A} \\
0, \\
\text { otherwise }
\end{array}\right.
$$

This interaction potential involves two parameters, $\epsilon_{W C A}$ and $\sigma_{W C A}$. The values of these parameters are taken as $\epsilon_{W C A}=k_{B} T$ and $\sigma_{W C A}=a_{0}$. The steepness of this potential at short distance requires to divide the streaming step into 200 intermediate MD steps, i.e. $\delta t_{M D}=0.005 \delta t_{c}$, thereby significantly increasing the computional time.

\subsection{Choice of the SRD parameters}

In order to perform a relevant comparison between the PNP prediction and SRD results, the SRD parameters are chosen to ensure that, for at least some of the systems under study, (i) electrostatic interactions are such that the density profile $c_{+}(z)$ is similar in SRD and within PB theory, (ii) the Reynolds number is small so that the Stokes equation is valid.

For all the SRD simulations, we chose $a_{0}=0.1 \mathrm{~nm}$ (thus $L_{\mathrm{hyd}}=45 a_{0}$ and $\left.l_{B-\mathrm{SRD}}=7.14 a_{0}\right)$. The length of the simulation box in the $x$ and $y$ directions is $36 a_{0}$. The average number of SRD particle per SRD collision cell is $\gamma=5$ and the time between two collisions is $\delta t_{c}=0.1 t_{0}$. The collision angle is $\alpha=130^{\circ}$. Using this choice of parameters, we ensure that we have a sufficiently high Schmidt number (ratio of diffusive momentum transfer over the diffusive mass transfer), $S c=\nu / D_{f}$, i.e. a liquid-like behaviour (see e.g. Ref. [27]). The viscosity of the SRD fluid can be computed using the analytical formula provided in Ref. [38]. This reduces in the present case to $\eta_{\mathrm{SRD}}=4.04 m_{f} \cdot a_{0}^{-1} \cdot t_{0}^{-1}$. Mapping the kinematic viscosity of the SRD fluid to that of pure water $\left(10^{-6} \mathrm{~m}^{2} . \mathrm{s}^{-1}\right)$, leads to the value of $t_{0}$ of $8.09 \mathrm{fs}$. The value of $m_{f}$ can be deduced from the values of $t_{0}$ and $a_{0}: m_{f}=t_{0}^{2} k_{B} T / a_{0}^{2}=2.710^{-23} \mathrm{~kg}$. The mass of the ions is $M=5 m_{f}$. This choice corresponds to $M / m_{F}=\gamma$, as prescribed by Ripoll and coworkers (see the discussion in [37]). As the mass of the ions determines its diffusion time scale, we should check that the ratio between this time scale and the convective transport time scale, i.e the Peclet number for the ions, is in the right regime. This number is given by:

$$
P e=\frac{v^{\circ} a_{\text {ion }}}{D_{\text {ion }}}
$$

if we consider a typical real system containing ions of diffusion coefficient $D_{\text {ion }}=$ $2.10^{-9} \mathrm{~m}^{2} \cdot \mathrm{s}^{-1}$, and size $a_{\text {ion }}=0.1 \mathrm{~nm}$, with an electric field of $E=10 \mathrm{~V} \cdot \mathrm{m}^{-1}$, the Peclet number is of the order of $10^{-6}$. It means we are in the regime where the ions diffuse very fast compared to the convective transport of the fluid. In our simulation, $D_{\text {ion }}=4.510^{-2} a_{0}^{2} \cdot t_{0}^{-1}$. Setting the electric field determines the characteristic velocity of the solvent, and therefore both the Peclet and the Reynolds 
numbers. Chosing $e E_{\mathrm{SRD}}=0.1 a_{0}^{-2} \cdot t_{0}^{-2}$, the Reynolds number corresponding to the reference velocity (Eq. 3) is then equal to $R e=0.03$, and the Peclet number is equal to $P e=0.01$, both in the required regime.

Chosing SRD parameters within the range of validity of the Poisson-Boltzmann theory is not straightforward. In principle, the best strategy would consist in decreasing the charge of the walls until the density profiles of PB and SRD perfectly match. But, as the length of the simulation box is limited by the computational costs, decreasing the charge density of the walls leads to the decrease of the number of counterions in the simulation box, hence an increase in the required simulation time to achieve the convergence of the counterion density and velocity profiles. The simulation parameters are thus chosen so as to obtain a good agreement between the PB and SRD profiles. More precisely, for the least charged system chosen (surface charge density $\sigma e$ of $-0.2 \mathrm{e} . \mathrm{nm}^{-2}$, with only 4 counterions in the simulation box), the difference between the counterion density profiles $c_{+}(z)$ is very small, (see Fig. 2). More importantly, for this system, the relative difference in the maximum of the solvent velocity profile $u_{x}(z)$ predicted by equation 1 is smaller than $2 \%$. Such a difference is sufficiently small to test the validity of the SRD methodology.

Another goal of the present study is to investigate how the velocity profiles deduced from SRD deviate from PNP predictions when the charge of the walls increases. To that end, we simulated systems with $\sigma e=-0.2,-0.5,-1,-2$ and -4 e.nm ${ }^{-2}$.

Finally, two additional parameters are varied, (i) the distance $L_{\mathrm{el}}$ characterizing the space available to counterions $\left(L_{\mathrm{el}}=4.3,4.4 \mathrm{or} 4.5 \mathrm{~nm}\right)$ and (ii) the discretization level $d$ of the charge within the solid. More precisely, each unit charge $-e$ on the solid wall can be divided among $d$ sites, each with a charge $-e / d$, located at a depth $w=0.1 \mathrm{~nm}$ (see Fig. 1). Increasing $d$ from 1 to 26, we decrease the depth of the electrostatic energy well experienced by the counterions on the surface, while maintaining the total charge of the surface constant. The sites are randomly placed, with the only constraint that the charges cannot get closer than half the average distance $1 / \sqrt{|\sigma|}$.

\begin{tabular}{|c|c|c|c|c|c|}
\hline & $\sigma=-0.2$ & $\sigma=-0.5$ & $\sigma=-1$ & $\sigma=-2$ & $\sigma=-4$ \\
\hline$L_{\mathrm{el}}=4.3$ & $d=26,6,1$ & $d=8,1$ & $d=4,1$ & $d=1$ & $d=1$ \\
\hline$L_{\mathrm{el}}=4.4$ & $d=26,13,6,3,1$ & $d=8,4,2,1$ & $d=4,2,1$ & $d=2,1$ & $d=1$ \\
\hline$L_{\mathrm{el}}=4.5$ & $d=26,6,1$ & $d=8,2,1$ & $d=4,2,1$ & $d=2,1$ & $d=1$ \\
\hline
\end{tabular}

Table 1. Simulated systems. $-e / d$ is the charge of the sites within the solid walls, i.e. each elementary charge is divided into $d$ sites on the wall, $L_{\mathrm{el}}$ is the width of the space available to the center of the counterions between the walls (in $\mathrm{nm}$ ) and $\sigma$ is the average charge density of the walls (in e.nm ${ }^{-2}$ ).

We did not perform all possible combinations of the different parameters. The systems for the SRD simulations without WCA force between the ions are summarized in Table 1 . For each system, the total simulation time is $10^{10} t_{0}$. For the simulations with WCA repulsion between the ions, we only considered the cases for which $d=1$, and $L_{\mathrm{el}}=4.4 \mathrm{~nm}$. For each system, the total simulation time is $4.10^{8} t_{0}$.

\section{Results}

\subsection{The SRD algorithm is able to describe electro-osmosis}

The behaviour of the SRD fluid and of the coupled solutes at low charge of the wall can be used to check the ability of the SRD methodology to describe electro- 
omosis. In Fig. 2, the density profiles of counterions are shown for the system with the smallest surface charge density $\left(\sigma e=0.2{\mathrm{e} . \mathrm{nm}^{-2}}^{-}\right)$with $L_{\mathrm{el}}=4.4 \mathrm{~nm}$. The corresponding solvent velocity profiles are shown in Fig. 3.

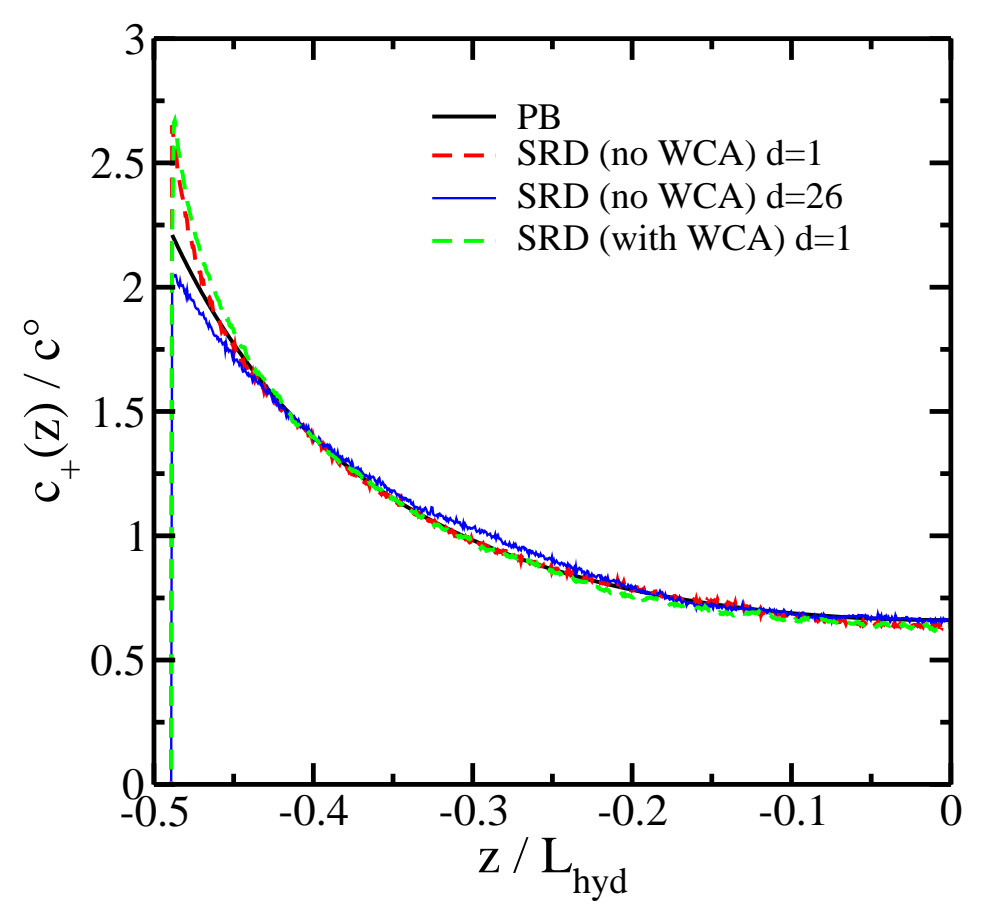

Figure 2. Conunterion density profile $c_{+}(z)$ as a function of the distance to the walls, for the systems with the lowest wall charge density $\left(\sigma e=-0.2\right.$ e. $\left.\mathrm{nm}^{-2}\right)$. The concentration is and averaged over the $x$ and $y$ directions. PB refers to Poisson-Boltzmann theory. The SRD results are shown for two values of the discretizaton parameter $d . c^{\circ}=2|\sigma| / L_{e l}$ is the average counterion concentration.

Since this system corresponds to the smallest number of counterions, the noise is more pronounced than with other systems. Nevertheless, the velocity profile obtained using SRD is remarkably close to the PNP result, and to the profiles obtained using the simulated counterion density $c_{+}(z)$ (cf. Eq. 1). Note that there is a partial slip at the fluid-solid boundary in SRD simulations, which was already observed with Poiseuille flow [42], but the velocity at the wall is small compared to the maximum velocity. In the absence of excluded volume between ions (no WCA), the relative difference between SRD and PNP is always smaller than $4 \%$, with the exception of the regions close to the boundaries (between $-0.5 L_{\mathrm{hyd}}$ and $\left.-0.4 L_{\mathrm{el}}\right)$ where partial slip occurs. These results validate the present algorithm for the simulation of electro-osmosis. In the presence of excluded volume between ions (SRD with WCA) the statistical noise is larger due to the shorter duration of the trajectory. Since the PNP theory does not account for this short-range repulsion, it should not be considered as a benchmark. We note however that the effect of ion-ion short-range repulsion is to decrease the fluid velocity, a behavior that is also observed for the other systems as we will show below. 


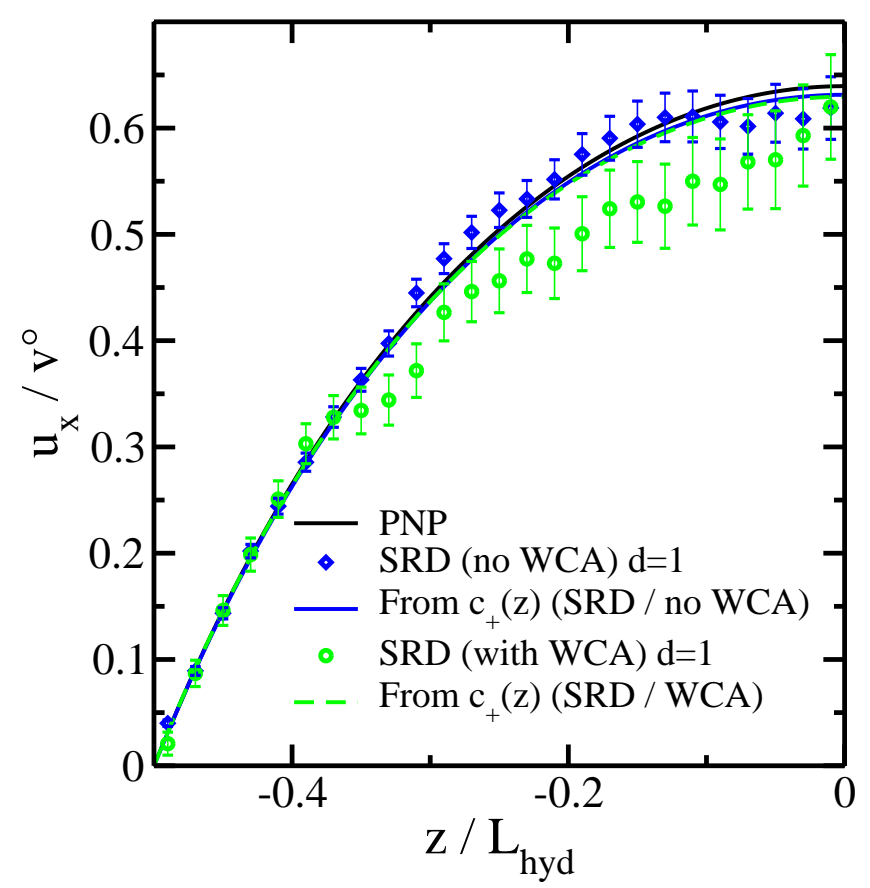

Figure 3. Solvent velocity field $u_{x}(z)$ in the direction of the electric field, averaged in the $x-y$ plane. PNP stands for Poisson-Nernst Planck theory. The SRD results are shown for two values of the discretizaton parameter $d$. For the SRD simulations, the profile obtained by integrating twice the counterion density $c_{+}(z)$ is also shown (see Eq. 1).

\subsection{Beyond mean-field with SRD}

When the average charge of the walls increases, the differences between the counterion density profiles predicted by the Poisson-Boltzmann treatment and the ones obtained through simulations become significant. All simulation results correspond to a discretization parameter $d=1$ and an effective ion-wall exclusion diameter $\sigma_{w}=1 \mathrm{~nm}\left(L_{\mathrm{el}}=4.4 \mathrm{~nm}\right)$. Similar results are obtained for $\sigma_{w}=2 \mathrm{~nm}$ $\left(L_{\mathrm{el}}=4.3 \mathrm{~nm}\right)$, while for $\sigma_{w}=0 \mathrm{~nm}\left(L_{\mathrm{el}}=4.5 \mathrm{~nm}\right)$ the discretization of the surface charge plays an essential role. The latter will be discussed in the next section and is not considered here.

Fig. 4 shows the counterion density profiles for the system with the highest charge $\left(\sigma e=-4\right.$ e.nm $\left.{ }^{-2}\right)$. While PB theory underestimates the ionic condensation at the surface of the solid, adding a short-range WCA repulsion does not affect the structure of the counterion fluid. In this regime, the structure is largely influenced by interionic correlations not captured by the mean-field treatment of PB. As the surface charge density increases, the differences between PB and SRD results become more pronounced, as expected. As the average distance between ions at the surface decreases, from $\approx 15 \sigma_{W C A}$ to $\approx 3 \sigma_{W C A}$ from $\sigma e=-0.5{\mathrm{e} . \mathrm{nm}^{-2}}^{-2}$ to -4 e.nm ${ }^{-2}$, electrostatic correlations increase and excluded volume starts to come into play. Nevertheless, the latter effect on the counterion density profiles is very limited.

The velocity profiles for three charge densities are shown in Fig. 5. First, as expected, larger differences in the counterion density profiles are transferred to the corresponding velocities obtained by Eq. 1. These differences are significant: For 


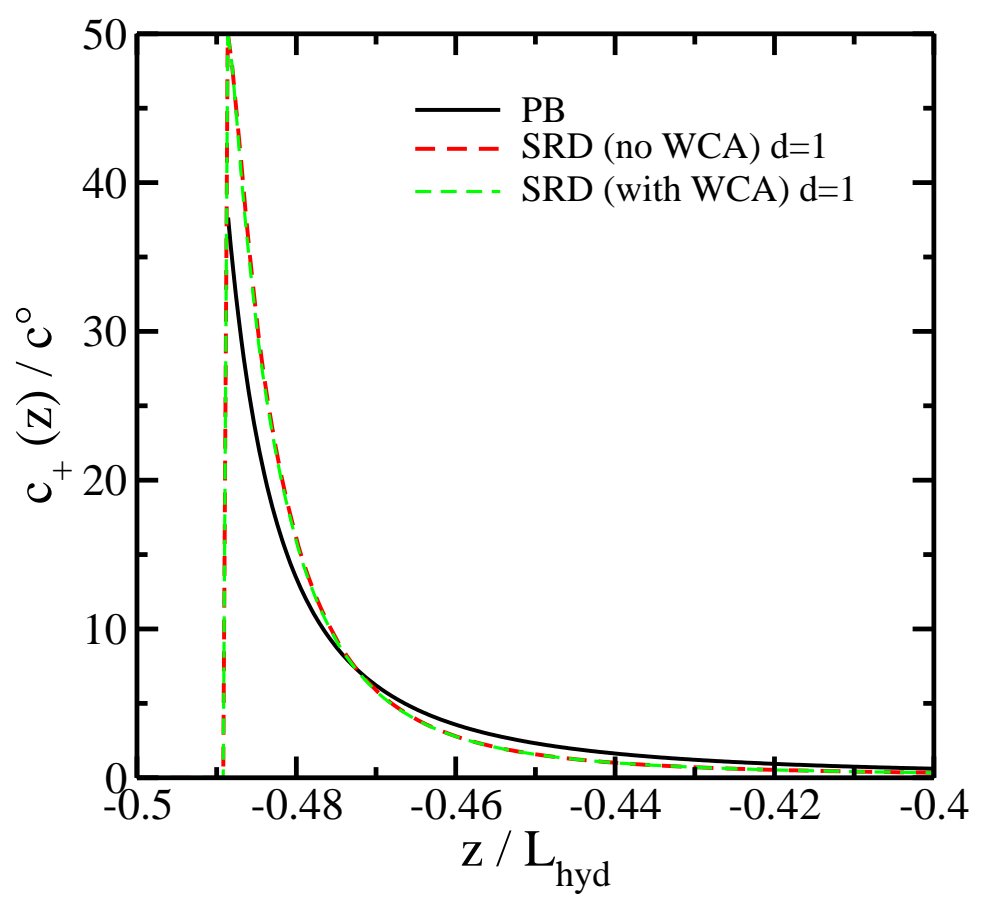

Figure 4. Couterion density profile $c_{+}(z)$ as a function of the distance from the wall, for the largest surface charge density $\sigma e=-4$ e.nm ${ }^{-2}, L_{\mathrm{el}}=4.4 \mathrm{~nm}$ and a discretization parameter $d=1$. PB refers to Poisson-Boltzmann theory. $c^{\circ}=2|\sigma| / L_{e l}$ is the average counterion concentration.

the most charged case, the maximum velocities differs by $\approx 34 \%$ from that obtained by SRD. Second, without short-range WCA repulsion, the SRD results are very

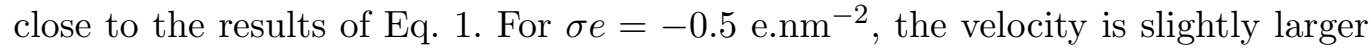
than expected. This may be due to the partial slip observed at the boundary. Indeed, despite the statistical uncertainty near the boundary, the extrapolated solvent velocity at the fluid/solid boundary does not vanish.

Nevertheless, the overall agreement is good for these systems. This suggests that Eq. 1 may apply even in some cases where in-plane correlations are present, as was already observed e.g. in Molecular Dynamics simulations [43, 44]. In addition, this indicates that SRD predicts the same behaviour as the Stokes equation (note that the transient regime, not analyzed here, may differ). Even though some compensations of errors are still possible, these results show that SRD may be used to simulate electrokinetic effects - and more generally to study fluid transport in the low Reynolds number limit.

The most dramatic differences are observed for the velocity profiles obtained with SRD in the presence of short range WCA repulsion between ions. While in the considered cases it has only little effect on the counterion density profiles (hence on the velocity profile predicted by Eq. 1), introducing this repulsion significantly reduces the overall electro-osmotic flow. As mentioned above, such interactions require the use of a much smaller time step, so that the simulated physical time is shorter than the one achievable without WCA repulsion between ions. However, this does not prevent us from reaching the stationary state even in that case, as illustrated in Fig. 6.

In fact, the effect of ion-ion correlations and that of the substrate charge distribu- 
(a)

(b)

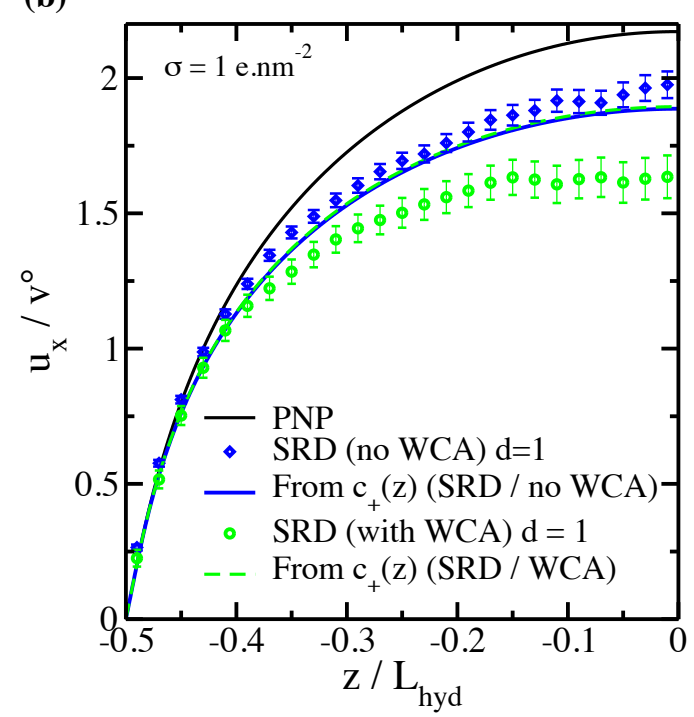

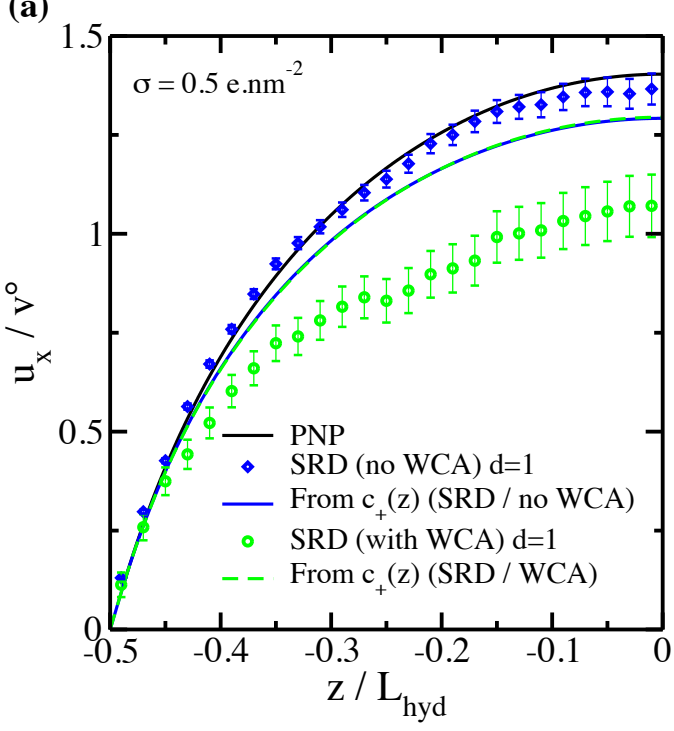

(c)

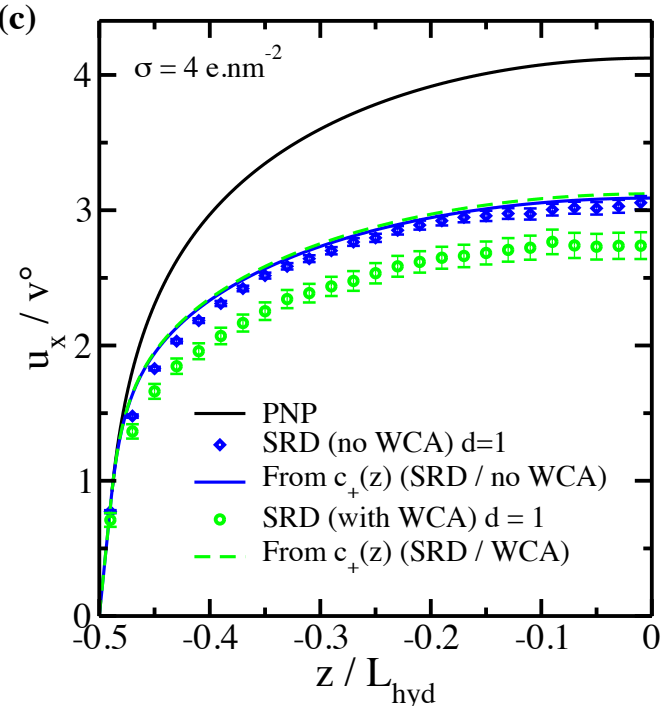

Figure 5. Solvent velocity profile $u_{x}(z)$ in the direction of the electric field, averaged in the $x-y$ plane, for three charge densities of the walls. PNP stands for Poisson-Nernst Planck theory. For the SRD simulations, the profile obtained by integrating twice the counterion density $c_{+}(z)$ is also shown (see Eq. 1).

tion and corrugation on the counterion distribution has already been investigated by Netz to discuss the consequences on electrokinetic effects, without accounting explicitely for hydrodynamics [45]. In the next sections, we finally investigate how the surface charge localization on discrete sites and the surface charge heterogeneity influence the electro-osmotic flow.

\subsection{Effect of surface charge localization}

For a given surface charge density, we tune the charge distribution inside the solid via the discretization parameter $d$ (see Fig. 1). Another control parameter which was not varied in the present study is the depth $w=0.1 \mathrm{~nm}$ within the solid of the charged sites. The strongest influence of the discretization is observed when the ions can approach closest to the wall, i.e. $\sigma_{w}=0\left(L_{\mathrm{el}}=L_{\mathrm{hyd}}=4.5 \mathrm{~nm}\right)$ and we focus here on this case. The electrostatic energy between a counterion and a surface site is then at most $-\left(l_{B} / w d\right) k_{B} T \approx-(7.14 / d) k_{B} T$. In principle, increasing $d$ should 


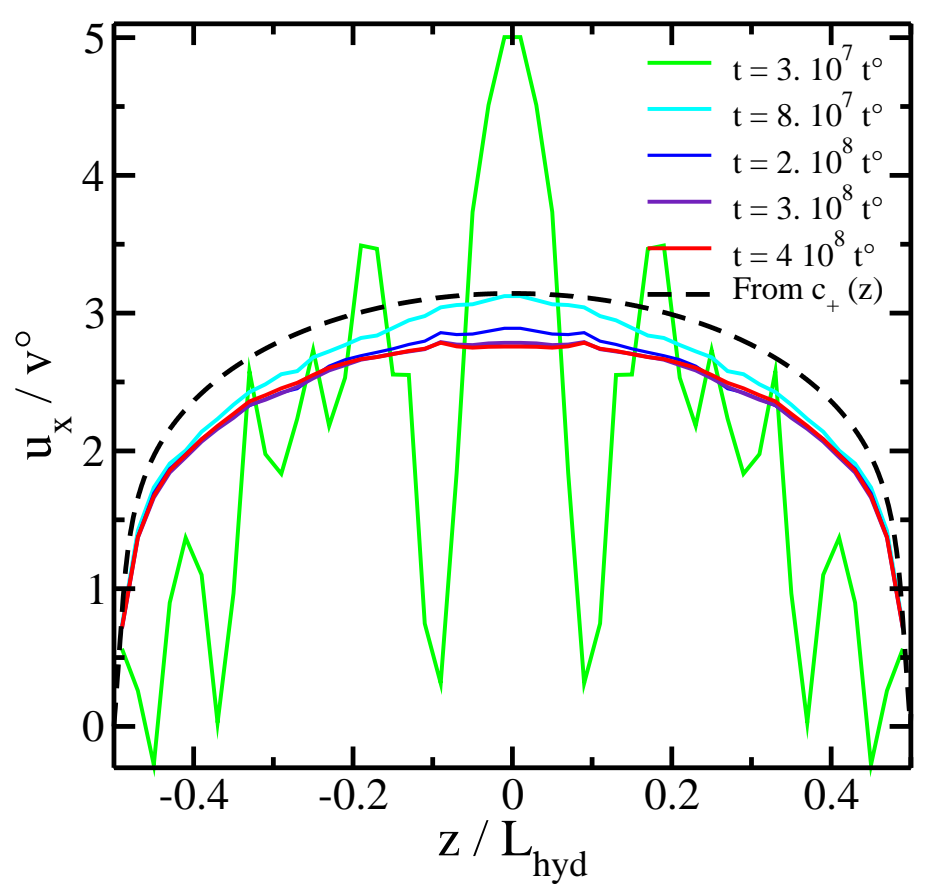

Figure 6. Solvent velocity profile $u_{x}(z)$ in the direction of the electric field, averaged in the $x-y$ plane, for $\sigma=4$ e.nm ${ }^{-2}$ and $L_{e l}=4.4 \mathrm{~nm}$, in the presence of WCA repulsion between ions. The different curves illustrate averages over different simulation times, from $3.10^{7} t_{0}$ to $4.10^{8} t_{0}$.

therefore smooth the interaction with the surface until the behaviour for a uniform continuous distribution is recovered (in that case, the depth $w$ is irrelevant). For this analysis, we use the computationally more efficient SRD method without WCA interactions.

The results are shown in Fig. 7 for two different surface charge densities $\sigma e=-0.5$ and -1 e.nm ${ }^{-2}$. As the discretization parameter changes, so does the counterion density profile $c_{+}(z)$ (not shown) and consequently the corresponding velocity profile obtained using Eq. 1. These predictions qualitatively follow the SRD results for the velocity profiles: The more localized the charge (i.e. the smaller $d$ is), the larger the decrease in the electro-osmotic flow. Moreover, the effect of $d$ on the flow is more pronounced for the smaller charge density (smaller number of counterions in the simulation box). We also note that as $\sigma$ (hence the number of counterions) increases, the predictions of Eq. 1 are in better agreement with the SRD velocity profile. Finally, we note that the PNP prediction may deviate signficantly (and not even systematically) from the simulation results in these regimes.

The results of the present section, where the ions interact more strongly with the charged surface sites due to a smaller distance to the charged surface sites, have several implications. On the one hand, they underline the limitations of the PNP approach with continuous surface charge distributions for systems in which the charge is indeed localized. Such a situation is in fact the rule rather than the exception, as in real systems the surface charge arises from charge defects in the solid or from the dissociation of surface sites. As is Molecular Dynamics, the present SRD strategy is capable of dealing with this issue. On the other hand, the above discussion indicates that the parameters describing the charge distribution 

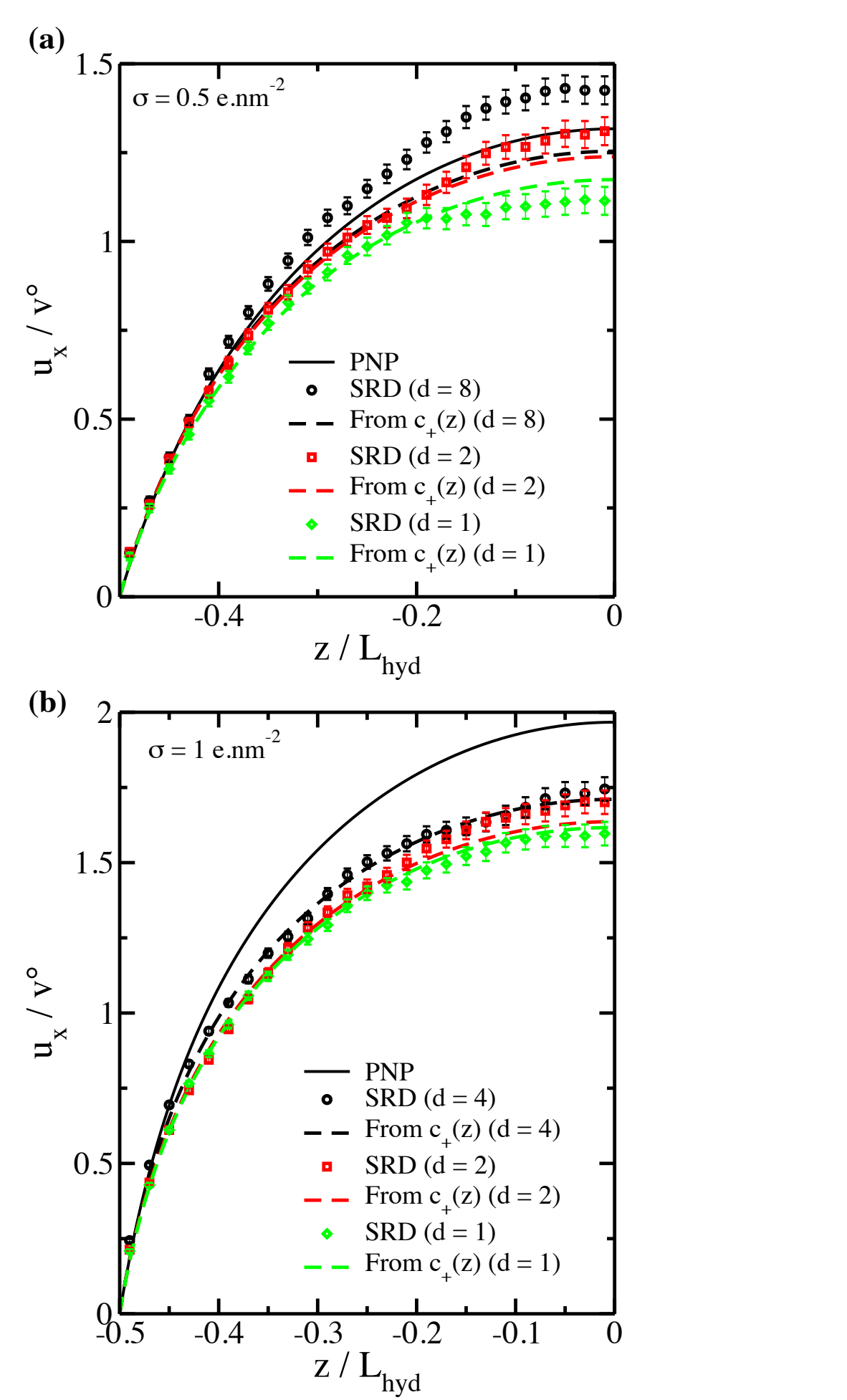

Figure 7. Solvent velocity profiles $u_{x}(z)$ in the direction of the electric field, for surface charge densities, $\sigma e=-0.5$ and -1 e.nm ${ }^{-2}, L_{\mathrm{el}}=4.5 \mathrm{~nm}$ and various values of the discretization parameter $d$.

in the SRD simulation (discretization parameter $d$, depth within the solid $w$ ) must be chosen carefully to describe a given physical system. Even though this was not done in the present study, it would also be useful to perform an average, for given simulation parameters, over several configurations of the charged sites.

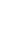

(1)

oe $=-0.5$ and -1 e.

\begin{abstract}
w) must
was not
for given
\end{abstract}

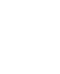

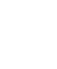




\subsection{Effect of surface charge heterogeneity}

Finally, in order to gain more insights into the effect of charge heterogeneity, we use the setup described in Fig. 8 to disentangle the contribution of the localization of the surface charge on discrete sites, discussed above, and the bare effect of the charge distribution. Indeed, the solid/liquid friction at the wall, which reduces the overall electro-osmotic flow, depends on the molecular nature of the fluid and the corrugation of the wall [45-47]. In the present SRD approach the charge is distributed over discrete surface sites and the resulting surface charge heterogeneity may in itself induce more complex electro-osmotic flows. Therefore, we consider as a further simplification the mean-field treatment of electrostatics of the PNP theory. As discussed above, several limitations of the PNP theory may be at the origin of deviations between the latter and the simulation results (see [23, 24, 43, 44] for comparison with other simulation methods). In order to address the effect of charge heterogeneity at the PNP level, we consider systems with the same average surface charge density and distance between the surfaces, with alternate charged and neutral stripes (see Figure 8) of equal width $L_{s}$. We investigate systematically the effect of this width on the total electro-osmotic flow in the presence of an electric field along the surfaces in the direction perpendicular to the stripes.

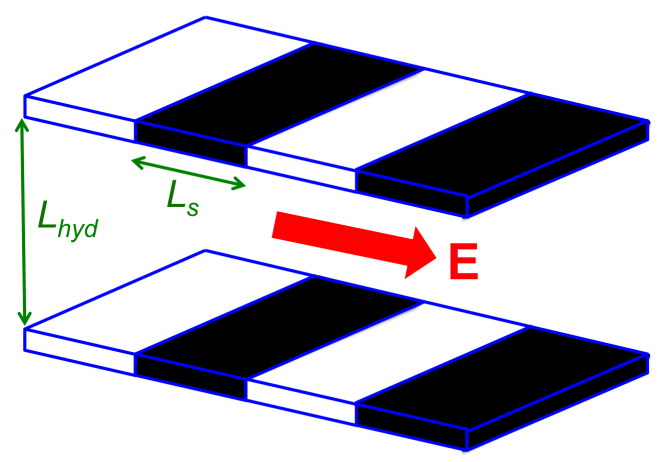

Figure 8. System simulated with LBE to investigate the effect of charge heterogeneity on the overall electro-osmotic flow. Surfaces are separated by a fixed distance $L_{h y d}$ and the average surface charge density $\sigma$ is distributed over alternate charged and neutral stripes of width $L_{s}$. The electric field $E$ is applied along the surface in the direction perpendicular to the stripes.

Since no analytical solution is available in that case, we use a lattice-based simulation method, which couples a Lattice-Boltzmann scheme for the enforcement of mass and momentum conservation to a time-dependent density functional framework for the evolution of the local composition and the corresponding local forces acting on the fluid. Using only the ideal term and mean-field electrostatics in the density functional, this Lattice-Boltzmann Electrokinetics (LBE) algorithm allows to describe electrokinetic effects at the level of the PNP theory [31]. It has already been validated for a range of problems similar to the one considered here and we refer the reader to previous works for the description of the method [32-34].

We use a lattice spacing $\Delta x=0.1 \mathrm{~nm}$. The distance between surfaces is $L_{h y d}=4.4 \mathrm{~nm}$ and the average surface charge density is $\sigma=-0.46296$ e.nm ${ }^{-2}$. The width of the stripes $L_{s}$ varies between 0 (homogeneous distribution) to $20 \mathrm{~nm}$. The diffusion coefficient of the counterions is $D_{+}=2.10^{-9} \mathrm{~m}^{2} \cdot \mathrm{s}^{-1}$ and the electric field is $E=14 \mathrm{~V} \cdot \mathrm{m}^{-1}$. All other physical parameters (viscosity, permittivity) correspond to water at room temperature. While the LBE method correctly captures the transient regime, we only consider here the stationary state. In practice, a simulation of $\approx 7.10^{4}$ time steps $(\approx 120 \mathrm{ps})$ is always sufficient to achieve the 
stationary state in the present cases.

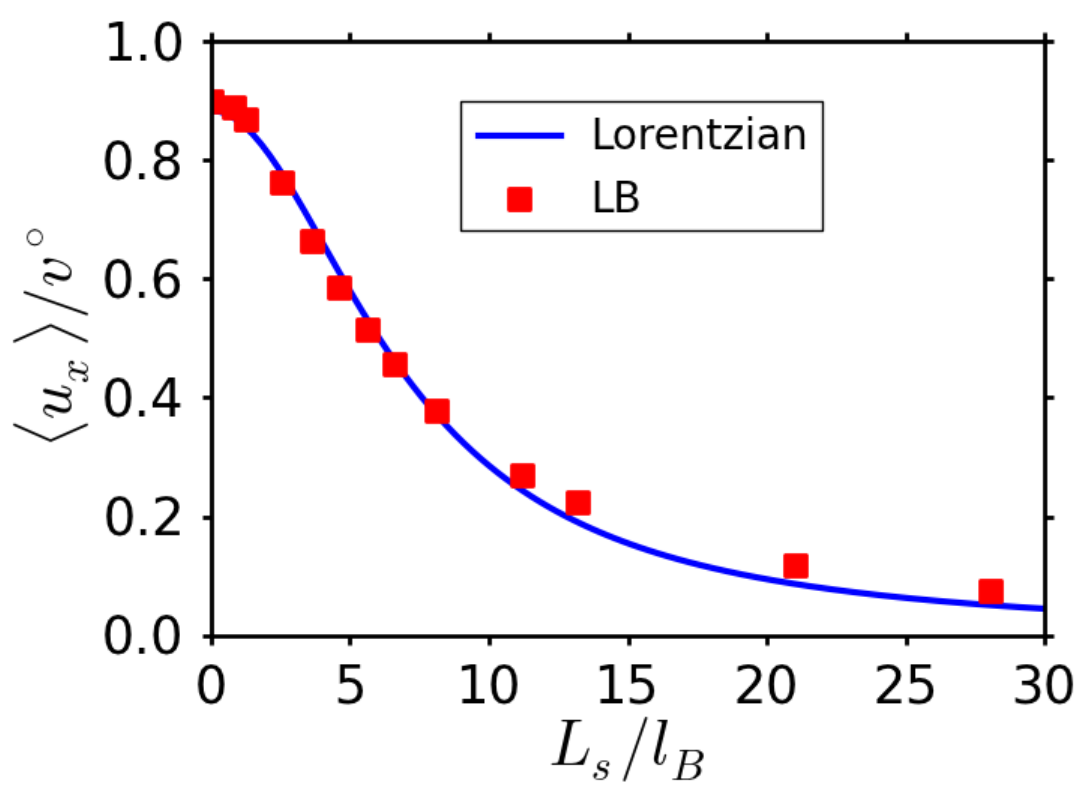

Figure 9. Average electro-osmotic velocity $\left\langle u_{x}\right\rangle$ for the striped charge distribution (see Fig. 8), as a function of the stripe width $L_{s}$ (normalized by the Bjerrum length $l_{B}$ ). Results are normalized by the reference velocity $v^{\circ}=e E / 2 \pi \eta l_{B}$.

The average electro-osmotic flow $\left\langle u_{x}\right\rangle$ for $L_{h y d}=4.4 \mathrm{~nm}$ and $\sigma=0.46296 e . \mathrm{nm}^{-2}$ is reported as a function of the stripe width $L_{s}$ in Fig. 9. Even at the mean-field level and with smooth charge distributions, the electro-osmotic flow can be dramatically reduced by the heterogeneity of the surface charge. This can be understood as the result of the heterogeneity of the counterion distribution, hence of the force in the presence of the applied electric field. This in turn induces a heterogeneous velocity profile (not shown). The more heterogeneous the velocity, the larger the dissipation by viscous stresses. As a result, a smaller fraction of the electric energy injected in the system is converted into the collective translation of the fluid. More quantitatively, the results are well described by a Lorentzian $A /\left[\left(L_{s} / l_{B}\right)^{2}+B^{2}\right]$. However the constants $A$ and $B$ may depend on the surface charge density and the distance $L_{h y d}$ between the surfaces. Since a systematic study is out of the scope of the present work, we did not investigate further the origin of this scaling with $L_{s}$.

\section{Conclusion}

Stochastic Rotation Dynamics, using the collisional coupling approach to capture momentum transfer between the semi-implicit solvent and the explicit counterions, is able to describe electro-kinetic effects in the limit of small Reynolds number. We have first validated the method for electro-osmosis in the simple case of a slit pore without added salt, for which an analytical solution of the Helmholtz-Smoluchowski theory (the steady-state solution of the Poisson-Nernst-Planck model) is known, in a physical regime where this mean-field theory applies.

We have then discussed the predictions of SRD for electro-osmosis beyond the range of validity of the PNP model. In particular, deviations from the mean-field 
predictions are observed for large surface charge densities arising from ion-ion correlations due to Coulomb and, as the average distance between ions at the surface decreases, excluded volume interactions. We analyzed the effect of the charge localization at the solid surface and of the charge heterogeneity, that both contribute to a reduction of the electro-osmotic flow. In order to disentangle these two aspects, we also investigated at the mean-field level a simple system with alternate charged and neutral stripes, using Lattice-Boltzmann Electrokinetics as a numerical solver of the PNP equations.

Overall, this work opens new perspectives for the use of SRD as a generic mesoscopic simulation method for soft matter problems, in particular under confinement, since in practice many interfaces between fluids and solids are charged. In that respect, the ability to tune the distribution of charges within the solid and the possibility to account for short-range ion-ion repulsion offer some flexibility to taylor the interactions at the surface to specific systems. It is now necessary to go beyond the case without added salt, considered here for validation purposes, and to analyze systematically the predictions of SRD in more complex geometries and the effect of ion-ion correlations on electro-kinetic phenomena.

\section{Acknowledgements}

The authors would like express their gratitude to Jean-Pierre Hansen for his guidance and their pleasure to enjoy him as a colleague at UPMC. BR is particularly indebted to Jean-Pierre for his continuing scientific inspiration and friendship, from his first interactions with him as a visiting student in Cambridge to a shared office in Paris. This work was supported by French state funds managed by the Agence Nationale de la Recherche (ANR) within the Investissements d'Avenir programme under reference ANR-11-IDEX-0004-02, and more specifically within the framework of the Cluster of Excellence MATISSE Partial financial support of the Agence Nationale de la Recherche in the frame of the project Celadyct (ANR-12BS08-0017-01) is also gratefully acknowledged.

\section{References}

[1] A. Malevanets and R. Kapral, J. Chem. Phys. 110 (17), 8605 (1999).

[2] G. Gompper, T. Ihle, D. Kroll and R. Winkler, Advances in Polymer Science 221, 1 (2008).

[3] T. Ihle and D. Kroll, Phys. Rev. E 67 (6), 066705 (2003).

[4] T. Ihle and D. Kroll, Phys. Rev. E 67 (6), 066706 (2003).

[5] N. Kikuchi, C.M. Pooley, J.F. Ryder and J.M. Yeomans, J. Chem. Phys. 119 (12), 6388 (2003).

[6] T. Ihle, E. Tüzel and D. Kroll, Phys. Rev. E 70 (3), 035701 (2004).

[7] C.M. Pooley and J.M. Yeomans, J. Phys. Chem. B 109 (14), 6505 (2005).

[8] A. Malevanets and R. Kapral, J. Chem. Phys. 112 (16), 7260 (2000).

[9] J. Padding and A. Louis, Phys. Rev. E 74 (3), 031402 (2006).

[10] A. Tomilov, A. Videcoq, T. Chartier, T. Ala-Nissilä and I. Vattulainen, J. Chem. Phys. 137, 014503 (2012).

[11] M. Ripoll, R.G. Winkler and G. Gompper, Phys. Rev. Lett. 96 (18), 188302 (2006).

[12] E. Tüzel, G. Pan, T. Ihle and D.M. Kroll, EPL (Europhysics Letters) 80 (4), 40010 (2007).

[13] H. Noguchi and G. Gompper, Phys. Rev. E 72 (1), 011901 (2005).

[14] R. Matthews and C.N. Likos, J. Phys. Chem. B 117 (27), 8283 (2013).

[15] J.K. Whitmer and E. Luijten, Journal of Physics: Condensed Matter 22 (10), 104106 (2010).

[16] A. Wysocki, C.P. Royall, R.G. Winkler, G. Gompper, H. Tanaka, A. van Blaaderen and H. Löwen, Faraday Discussions 144, 245 (2010).

[17] S. Hanot, M. Belushkin and G. Foffi, Faraday Discussions 9, 291 (2013). 
[18] E.S. Boek, A.D. Wilson, J.T. Padding, T.F. Headen and J.P. Crawshaw, Energy \& Fuels 24 (4), 2361 (2010).

[19] E.S. Boek, T.F. Headen and J.T. Padding, Faraday Discussions 144, 271 (2010).

[20] A. Nikoubashman, C.N. Likos and G. Kahl, Soft Matter 9, 2603 (2013).

[21] H. Noguchi and G. Gompper, Proceedings of the National Academy of Sciences of the United States of America 102 (40), 14159 (2005).

[22] E. Westphal, S.P. Singh, C.C. Huang, G. Gompper and R.G. Winkler, Computer Physics Communications 185 (2), 495 (2014).

[23] I. Pagonabarraga, B. Rotenberg and D. Frenkel, Phys. Chem. Chem. Phys. 12 (33), 9566 (2010).

[24] B. Rotenberg and I. Pagonabarraga, Molecular Physics 111 (7), 827 (2013).

[25] S. Frank and R.G. Winkler, J. Chem. Phys. 131 (23), 234905 (2009).

[26] S.P. Singh, C.C. Huang, E. Westphal, G. Gompper and R.G. Winkler, J. Chem. Phys. 141 (8), 084901 (2014).

[27] G. Batôt, V. Dahirel, G. Mériguet, A.A. Louis and M. Jardat, Phys. Rev. E 88 (4), 043304 (2013).

[28] A. Chatterji and J. Horbach, J. Chem. Phys. 122, 184903 (2005).

[29] V. Lobaskin, B. Dünweg, M. Medebach, T. Palberg and C. Holm, Phys. Rev. Lett. 98 (17) (2007).

[30] K. Grass and C. Holm, Journal of Physics: Condensed Matter 20 (49), 494217 (2008).

[31] F. Capuani, I. Pagonabarraga and D. Frenkel, J. Chem. Phys. 121 (2), 973 (2004).

[32] B. Rotenberg, I. Pagonabarraga and D. Frenkel, EPL (Europhysics Letters) 83 (3), 34004 (2008).

[33] B. Rotenberg, I. Pagonabarraga and D. Frenkel, Faraday Discussions 144, 223 (2010).

[34] A. Obliger, M. Duvail, M. Jardat, D. Coelho, S. Békri and B. Rotenberg, Phys. Rev. E 88 (1), 013019 (2013).

[35] P. Warren, Int. J. Mod. Phys. C 8 (4), 889 (1997).

[36] T. Ihle and D.M. Kroll, Phys. Rev. E 63, 020201(R) (2001).

[37] M. Ripoll, K. Mussawisade, R.G. Winkler and G. Gompper, Phys. Rev. E 72, 016701 (2005).

[38] G. Gompper, T. Ihle, D. Kroll and R. Winkler, Multi-Particle Collision Dynamics: A ParticleBased Mesoscale Simulation Approach to the Hydrodynamics of Complex Fluids, in Advanced Computer Simulation Approaches for Soft Matter Sciences III, edited by Christian Holm and Kurt Kremer, Advances in Polymer Science, Vol. 221 (, , 2009), pp. 1-87.

[39] A. Lamura, G. Gompper, T. Ihle and D.M. Kroll, Europhys. Lett. 56, 319 (2001).

[40] P.S. Crozier, R.L. Rowley, E. Spohr and D. Henderson, J. Chem. Phys. 112 (21) (2000).

[41] J.D. Weeks, D. Chandler and H.C. Andersen, J. Chem. Phys. 54 (12), 5237 (1971).

[42] J.K. Whitmer and E. Luijten, J. Phys.: Condens. Matter 22, 104106 (2010).

[43] A. Botan, V. Marry, B. Rotenberg, P. Turq and B. Noetinger, J. Phys. Chem. C 117 (2), 978 (2013).

[44] A. Botan, V. Marry, B. Rotenberg, P. Turq and B. Noetinger, J. Phys. Chem. C 117 (39), 20376 (2013).

[45] R.R. Netz, Phys. Rev. Lett. 91 (13), 138101 (2003).

[46] L. Bocquet and J.L. Barrat, Phys. Rev. E 49 (4), 3079 (1994).

[47] K. Falk, F. Sedlmeier, L. Joly, R.R. Netz and L. Bocquet, Langmuir 28 (40), 14261 (2012). 